\title{
Autonomic Nervous System Response to Heat Stress Exposure by Means of Heart Rate Variability
}

\author{
Spyridon Kontaxis ${ }^{1}$, Raquel Bailón ${ }^{1,2}$, Andrius Rapalis ${ }^{3}$, Marius Brazaitis ${ }^{4}$, Margarita Cernych ${ }^{4}$ \\ Jesús Lázaro $^{5,1,2}$, Pablo Laguna ${ }^{1,2}$, Eduardo Gil ${ }^{1,2}$, Vaidotas Marozas ${ }^{3}$ \\ ${ }^{1}$ BSICoS Group, I3A, IIS Aragón, University of Zaragoza, Spain \\ ${ }^{2}$ CIBER de Bioingeniería, Biomateriales y Nanomedicina, Spain \\ ${ }^{3}$ Biomedical Engineering Institute, Kaunas University of Technology, Lithuania \\ ${ }^{4}$ Lithuanian Sports University, Institute of Sports Science and Innovation, Lithuania \\ ${ }^{5}$ Department of Electrical Engineering of University of Connecticut, USA
}

\begin{abstract}
In this study, a heart rate variability (HRV) analysis guided by respiration is conducted to assess changes in autonomic nervous system (ANS) regulation during pronounced heat stress induced by repetitive exposures to dry sauna in 13 young healthy subjects. The spectral power at the low frequency $(L F)$ band $[0.04,0.15] \mathrm{Hz}$ and at the high frequency band centered around the mean respiratory rate, estimated from ECG-derived respiration signals, are studied. The mean heart rate (HR), the total power of $H R V$, and the normalized LF power are also analyzed.

Results show that mean HR and normalized LF power increase significantly after basal stage, not only during the sauna sessions, but also at the intermediate short rest stages and recovery phase after $30 \mathrm{~min}$. A significant reduction in the total power of HRV during all sauna sessions is observed, while an increased power in LF band is shown only during recovery which might be related to improved cardiac function (increased cardiac output and reduced peripheral vascular resistance) after heat exposure. Respiratory rate does not change significantly during the protocol but a negative trend at stress stages may indicate a reverse reaction against an over-activation of the sympathetic branch. In conclusion, exposure to heat stress shifts sympathovagal balance of ANS toward sympathetic dominance that increases $H R$ and diminishes HRV.
\end{abstract}

\section{Introduction}

Heat stress, during exposure to elevated environmental temperatures and/or exercise, results in pronounced cardiovascular adjustments that are necessary for adequate temperature regulation [1]. Thermal signals originated from thermo-receptors in the skin are transmitted by neurons in the preoptic area of the hypothalamus where tran- sient receptor potential cation channels transduce skin temperature into primary thermoreceptor afferent neuronal activity that controls the autonomic thermoregulation [2].

An increment in body temperature, increases skin blood flow (cutaneous vasodilation) and part of blood volume is diverted from the internal organs to body peripheral parts, where heat transfer occurs in conjunction with sweating [3]. Cardiac output is increased, which is primarily mediated through increases in heart rate (HR), while cardiac stroke volume remain unchanged and minimal changes are observed for arterial blood pressure since there is an increased blood flow towards skin [4].

Autonomic nervous system (ANS) plays an important role in cardiovascular homeostasis through the fast and short-term regulation of HR. Heart rate variability (HRV) has been widely used for assessing the balance between the parasympathetic and sympathetic branch of the ANS, by means of spectral-domain indices which are based on the power ratio at low frequency band $(\mathrm{LF},[0.04,0.15] \mathrm{Hz})$ and high frequency band $(\mathrm{HF},[0.15,0.4] \mathrm{Hz})[5]$.

A variety of studies have analyzed the effects of passive (i.e., non-exercising) heat stress on HRV indices, involving protocols of immersion hydrotherapy, wet sauna, and dry sauna. However, few studies have investigated changes in sympathovagal balance at extremely hightemperature and dry environments in healthy subjects. Bruce et al. [6] reported that during a single sauna session HR and sympatho-vagal ratio increased significantly compared to pre-exposure. Zalewski et al. [7] reported an increment in HR and sympatho-vagal ratio immediately after the exposure that lasted up to 3 hours later.

The aim of this study is to investigate ANS responses to pronounced heat stress stimuli induced by repetitive exposures to dry sauna and the respective recovery profile 30 min after the last sauna bathing. An additional novelty of this study is the inclusion of respiratory information, esti- 
mated from an ECG-derived respiration (EDR) method, in HRV analysis [8], since none of the previous sauna studies have considered the potential influence of respiratory rate on spectral-domain indices of HRV.

\section{Materials and methods}

\subsection{Experimental protocol}

Thirteen healthy subjects (all men, mean \pm standard deviation age $24.61 \pm 4.13$ years, body mass index $24.43 \pm 3.17 \mathrm{~kg} / \mathrm{m}^{2}$ ) underwent a heat stress protocol which consists of repetitive exposures to sauna characterized by air with a relative humidity of $30 \%$ and high temperature $80-90{ }^{\circ} \mathrm{C}$. Before and after sauna exposures, participants were instructed to rest at semi Fowler's position in a neutral temperature environment $\left(25^{\circ} \mathrm{C}\right)$. The total duration of the protocol was approximately 2 hours and 20 minutes. The timeline of the study protocol is shown in Fig. 1. The experimental procedures were approved by the Lithuanian University of Health Sciences Kaunas Region Biomedical Research Ethics Committee (protocol number BE-2-9). The modified Einthoven leads of ECG (I, II, and III leads) and the chest skin temperature were recorded by using the Nautilus1 (BMII, Lithuania) at a sampling frequency of $1000 \mathrm{~Hz}$ and $10 \mathrm{~Hz}$, respectively.

\subsection{Preprocessing}

First, QRS-complexes are detected on lead II [9]. Then, ectopic beats and misdetections are corrected by using the heart timing signal [10]. The respiratory rate $f_{r}(t)$ is estimated from three single-lead EDR signals; the upand down-slope of the QRS complex [11], and the slope range [12]. EDR signals from different leads (lead II and III) are combined to increase the robustness of respiratory rate estimation from EDR spectra [12].

\subsection{HRV analysis}

HRV is defined based on the time-varying pulse integral frequency modulation model described in [13],

$$
m(t)=\frac{d_{\mathrm{HR}}(t)-d_{\mathrm{HRM}}(t)}{d_{\mathrm{HRM}}(t)},
$$

where $m(t)$ is the modulating signal which consists of the variations of the instantaneous $\mathrm{HR} d_{\mathrm{HR}}(t)$ around the mean HR $d_{\mathrm{HRM}}(t)$, obtained by low-pass filtering $d_{\mathrm{HR}}(t)$, plus a correction accounting for a time-varying mean HR.

Spectral indices are estimated from the power spectral density of $m(t), \quad S_{j}(f)$, obtained by applying Welch's periodogram (50-s Hamming windows with $50 \%$ overlap) every $60 \mathrm{~s}$ in segments of length 5 minutes. For the $j$ :th segment, the power at LF

\begin{tabular}{c}
\begin{tabular}{|c|c|c|c|c|c|c|c|c|c|}
\hline$R_{0}$ & $S_{1}$ & $R_{1}$ & $S_{2}$ & $R_{2}$ & $S_{3}$ & $R_{3}$ & $S_{4}$ & $R_{4}$ \\
$0: 00$ & $0: 20$ & $0: 35$ & $0: 50$ & $1: 00 \quad 1: 15$ & $1: 25$ & $1: 40$ & $1: 50$ & & $2: 20$ \\
time (h:mm)
\end{tabular} \\
\hline
\end{tabular}

Figure 1. Timeline of the study protocol. Rest stages $\left(R_{0}\right.$, $\left.R_{1}, R_{2}, R_{3}, R_{4}\right)$ and sauna sessions $\left(S_{1}, S_{2}, S_{3}, S_{4}\right)$ are marked in blue and red, respectively.

band, at the extended high-frequency band (eHF: $\left.\left[0.15, d_{\mathrm{HRM}}(j) / 2\right] \mathrm{Hz}\right)$, and at the high-frequency band centered around $f_{r}(j)$ (cHF: $\left[f_{l}(j), f_{u}(j)\right] \mathrm{Hz}$ ), where the lower limit is $f_{l}(j)=\max \left\{0.15, f_{r}(j)-0.15\right\}$ and the upper is $f_{u}(j)=\min \left\{f_{r}(j)+0.15, d_{\mathrm{HRM}}(j) / 2\right\}$, are estimated and denoted as $P_{\mathrm{LF}}(j), P_{\mathrm{HF}}^{e}(j)$, and $P_{\mathrm{HF}}^{c}(j)$, respectively. Based on the above, the total spectral power is given by $P_{\mathrm{TOT}}(j)=P_{\mathrm{LF}}(j)+P_{\mathrm{HF}}^{e}(j)$, while the normalized LF power is defined as

$$
P_{\mathrm{LFn}}^{c}(j)=P_{\mathrm{LF}}(j) /\left(P_{\mathrm{LF}}(j)+P_{\mathrm{HF}}^{c}(j)\right),
$$

where cHF band is slightly wider than in the original work [14]. Note that $d_{\mathrm{HRM}}(j)$ and $f_{r}(j)$ refer to the temporal mean value of the parameter in the $j$ :th segment.

\subsection{Statistical analysis}

To reduce the parameters variance, the average across segments for $10 \mathrm{~min}$ in each stage is used and the features are denoted as $\bar{T}, \bar{d}_{\mathrm{HRM}}, \bar{P}_{\mathrm{TOT}}, \bar{P}_{\mathrm{LF}}, \bar{P}_{\mathrm{HF}}^{c}$, and $\bar{P}_{\mathrm{LFn}}^{c}$. The 10 central minutes of the stages $R_{0}, R_{1}, R_{2}, R_{3}$, and $S_{1}$, while the whole stages $S_{2}, S_{3}$, and $S_{4}$ are subjected to analysis. Only the last 10 minutes of the recovery phase $R_{4}$ are analyzed in an attempt to characterize the late response. Besides HRV parameters, statistical analysis is also conducted for the mean skin temperature $\bar{T}$. It should be noted that segments where $\bar{f}_{r}(j) \leq 0.15 \mathrm{~Hz}$ are excluded for 2 subjects; $50 \%$ in $S_{4}$ for the first one, while for the second $16 \%, 50 \%$, and $33 \%$ in $R_{1}, R_{2}$, and $R_{3}$, respectively.

A paired Wilcoxon signed-rank test is applied after normality rejection (Kolmogorov-Smirnov test) in order to assess intra-subject differences in ANS regulation between $R_{0}$ and each stage of the protocol. Statistical differences are considered when significance level is $p<0.05$.

\section{Results}

Figure 2 illustrates the inter-subject mean and standard deviation for all the parameters used in HRV analysis during the whole study. Figure 3 shows an example of respiratory rate estimation from EDR signals. Table 1 summarizes the results of statistical analysis. Results show that both $\bar{T}$ and $\bar{d}_{\text {HRM }}$ increase significantly $(p<0.001)$ after basal stage $R_{0}$, not only during the sauna sessions $\left(S_{1}, S_{2}\right.$, $S_{3}$, and $\left.S_{4}\right)$ but also at the intermediate rest stages $\left(R_{1}, R_{2}\right.$, 
(a)

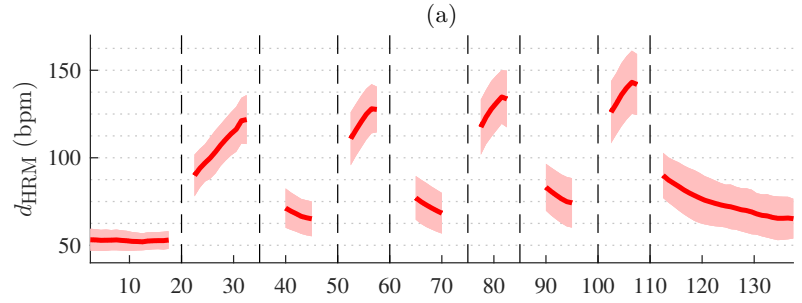

(c)

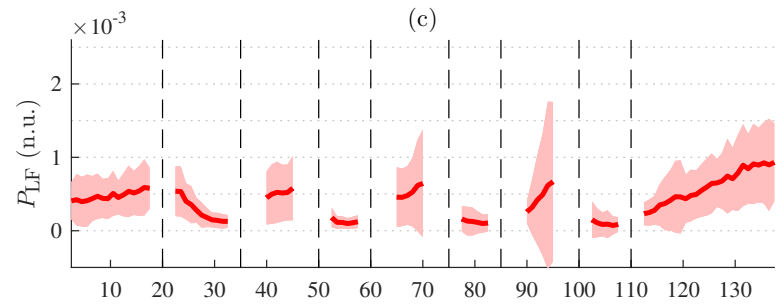

(e)

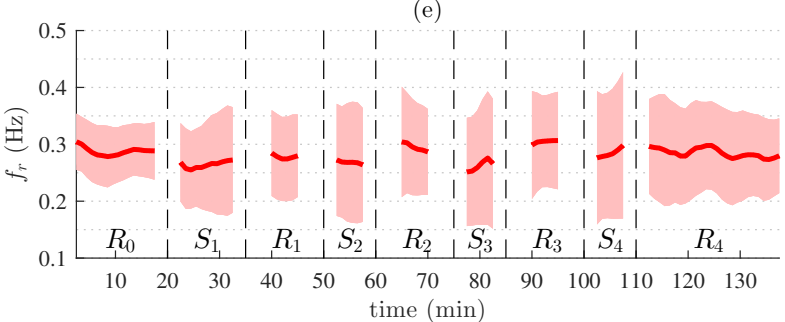

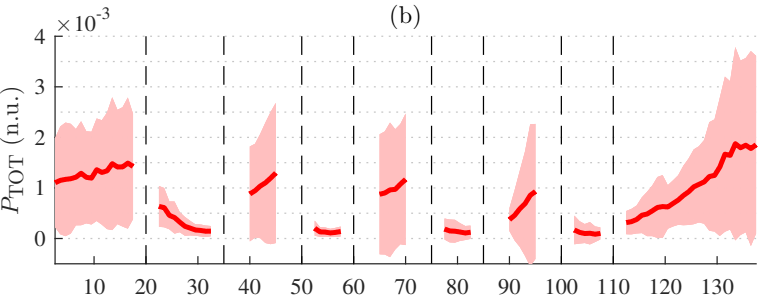

(d)

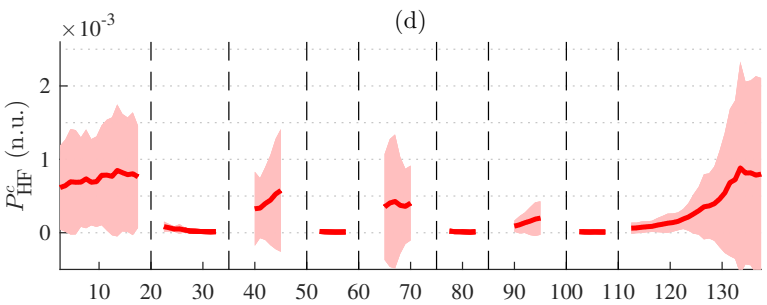

(f)

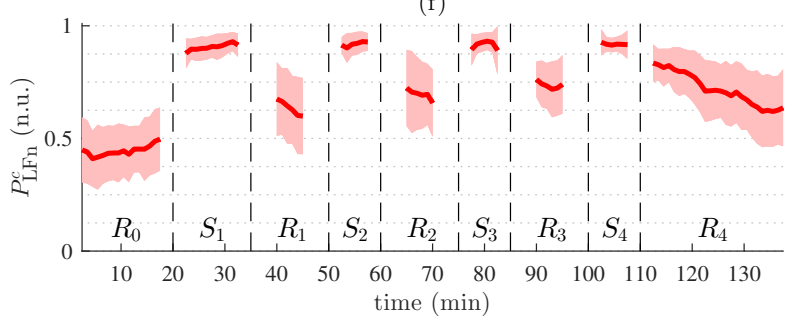

Figure 2. Inter-subject mean and standard deviation for all the parameters used in HRV analysis. (a) mean HR, (b) total HRV power, (c) power at LF band, (d) power at cHF band, (e) mean respiratory rate, (f) normalized LF power.

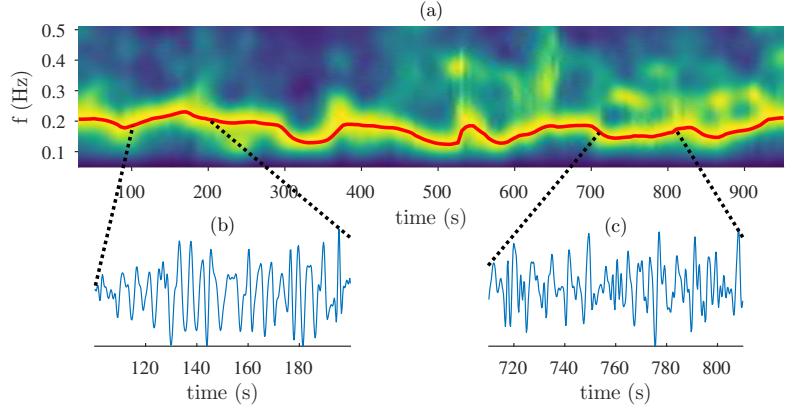

Figure 3. Respiratory rate estimation from EDR singals. (a) Time-frequency EDR spectrum, (b)-(c) excerpts of one EDR signal. The estimated respiratory rate is displayed with a red line.

and $R_{3}$ ), and recovery phase $R_{4}$. No statistically significant differences are obtained for $\bar{f}_{r}$, although lower median values are observed during stress stages in Fig. 4.

A significant reduction in $\bar{P}_{\mathrm{TOT}}, \bar{P}_{\mathrm{LF}}$, and $\bar{P}_{\mathrm{HF}}^{c}$ during all sauna sessions is observed, while an increased $\bar{P}_{\mathrm{LF}}$ is shown only during recovery. The values of $\bar{P}_{\mathrm{LFn}}^{c}$ increase significantly after $R_{0}$ for all the stages, as it occurs with HR. Note that $\bar{P}_{\text {TOT }}$ and $\bar{P}_{\mathrm{HF}}^{c}$ are also significantly lower before last sauna session $\left(R_{3}\right.$, with $p<0.05$ and $p<0.001$,

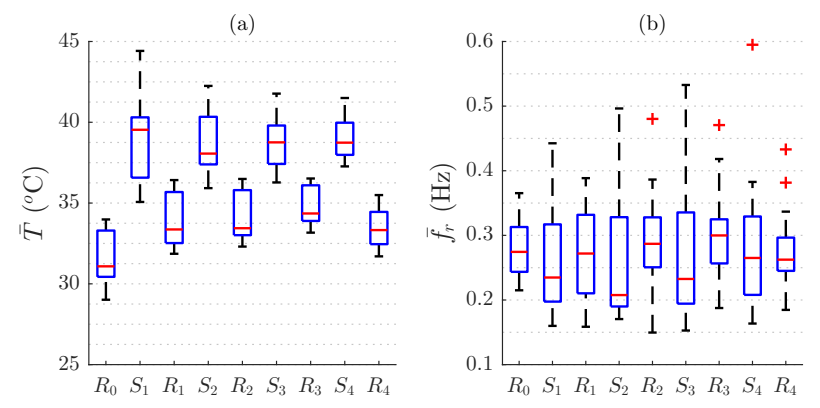

Figure 4. Boxplots of parameters used in statistical tests. The mean (a) skin temperature, (b) respiratory rate.

respectively). No significant differences are obtained for $\bar{P}_{\text {TOT }}$ neither at $R_{1}$ nor at $R_{2}$. However, $\bar{P}_{\mathrm{HF}}^{c}$ remains significantly reduced during both $R_{1}$ and $R_{2}$ compared to $R_{0}$ but with a lower significance level $(p<0.05)$ relative to that obtained in $R_{3}(p<0.001)$.

\section{Discussion}

In this study, a HRV analysis guided by respiration is conducted to assess changes in ANS regulation during pronounced passive heat stress multi-stage stimuli. 
Table 1. Results of statistical analysis. The values of the intra-subject average parameters are displayed as inter-subject median (interquartile range) and significant differences relative to $R_{0}$ are marked with ${ }^{*}(p<0.05)$ and ${ }^{* *}(p<0.001)$.

\begin{tabular}{|c|c|c|c|c|c|c|c|c|c|}
\hline & $R_{0}$ & $S_{1}$ & $R_{1}$ & $S_{2}$ & $R_{2}$ & $S_{3}$ & $R_{3}$ & $S_{4}$ & $R_{4}$ \\
\hline$T\left({ }^{o} \mathrm{C}\right)$ & $31.0(2.9)$ & $39.5(3.7)^{* *}$ & $33.7(3.1)^{* *}$ & $38.1(2.9)^{* *}$ & $33.4(2.8)^{* *}$ & $38.7(2.4)^{* *}$ & $34.3(2.2)^{* *}$ & $38.7(2.0)^{* *}$ & $33.3(2.0)^{* *}$ \\
\hline $\bar{d}_{\mathrm{HRM}}(\mathrm{bpm})$ & $50(8)$ & $104(13)^{* *}$ & $63(18)^{* *}$ & $118(17)^{* *}$ & $72(23)^{* *}$ & $121(20)^{* *}$ & $73(22)^{* *}$ & $133(21)^{* *}$ & $62(16)^{* *}$ \\
\hline $\bar{f}_{r}(\mathrm{~Hz})$ & $0.27(0.07)$ & $0.23(0.12)$ & $0.27(0.12)$ & $0.21(0.14)$ & $0.29(0.08)$ & $0.23(0.14)$ & $0.30(0.01)$ & $0.26(0.12)$ & $0.26(0.05)$ \\
\hline $\bar{P}_{\text {TOT }} \cdot 10^{5}$ (n.u.) & $80.2(125.0)$ & $24.8(28.3)^{* *}$ & $64.8(84.4)$ & $11.2(15.3)^{* *}$ & $58.2(53.8)$ & $7.6(13.1)^{* *}$ & $44.6(29.1)^{*}$ & $3.7(8.2)^{* *}$ & $134.0(128.0)$ \\
\hline $\bar{P}_{\mathrm{LF}} \cdot 10^{5}$ (n.u.) & $34.8(39.3)$ & $21.7(22.0)^{*}$ & $42.6(55.5)$ & $9.1(12.9)^{* *}$ & $31.4(26.4)$ & $6.7(10.6)^{* *}$ & $28.3(18.1)$ & $3.2(7.2)^{* *}$ & $95.3(62.7)^{*}$ \\
\hline $\bar{P}_{\mathrm{HF}}^{c} \cdot 10^{5}$ (n.u.) & $45.9(68.9)$ & $2.1(3.4)^{* *}$ & $19.4(34.4)^{*}$ & $0.9(1.8)^{* *}$ & $15.2(20.9)^{*}$ & $0.7(1.0)^{* *}$ & $11.3(11.7)^{* *}$ & $0.2(0.7)^{* *}$ & $32.0(44.7)$ \\
\hline $\bar{P}_{\mathrm{LFn}}^{c}$ (n.u.) & $0.45(0.12)$ & $0.91(0.01)^{* *}$ & $0.69(0.22)^{* *}$ & $0.93(0.06)^{* *}$ & $0.74(0.20)^{* *}$ & $0.92(0.07)^{* *}$ & $0.75(0.08)^{* *}$ & $0.92(0.06)^{* *}$ & $0.65(0.17)^{* *}$ \\
\hline
\end{tabular}

Exposure to repetitive dry sauna sessions alternated with a 15 minute period of rest causes to heat-stressed subjects an increment in $\bar{d}_{\mathrm{HRM}}, \bar{T}$, and $\bar{P}_{\mathrm{LFn}}^{c}$, during all stages compared to basal. In this study, the mean respiratory rate most of the times lies in the band $[0.15,0.4] \mathrm{Hz}$, and thus similar results (not shown) are obtained for the normalized LF power using either the classical HF band [5] or eHF band.

In [6], the exposure to single sauna session led to a minor warming intervention with negligible effects on physiological strain where HR was reported to be increased from 65 to $106 \mathrm{bpm}$, while in [7] no data were collected during the sauna. In this study, a higher increment of mean HR (up to $133 \mathrm{bpm}$ ) in combination with the elevated mean core body temperature (up to $38.7^{\circ} \mathrm{C}$ ) suggests that repetitive exposures to sauna sessions induces internal thermal strain level meeting the criteria of severe whole-body hyperthermia level.

While proceeding to the next sauna exposure, a positive trend from $S_{1}$ to $S_{4}$ is observed for $\bar{d}_{\mathrm{HRM}}$, while $\bar{P}_{\mathrm{HF}}^{c}$ follows a negative trend at the intermediate rest stages $R_{1}$, $R_{2}$, and $R_{3}$ (Table 1 ). The short duration of the rest intermediate stages do not allow full recovery, which in turn leads to higher stress of the body in the following sauna session. Lower respiratory rates during sauna (Fig. 4) may be a mechanism for avoiding a potential over-activation of the sympathetic branch. During recovery phase, a higher $\bar{P}_{\mathrm{LF}}$ (relative to $R_{0}$ ) might be related to improved cardiac function after heat exposure since it has been suggested that increased cardiac output and reduced peripheral vascular resistance are the main mechanisms of action for the health effects of frequent sauna bathing [15].

\section{Conclusions}

Spectral indices of HRV are able to quantify and monitor shifts of sympathovagal balance toward sympathetic dominance induced by exposure to heat stress multi-stage stimuli with short intermediate rest stages.

\section{Acknowledgment}

This work was supported by Ministerio de Economía y Competitividad (RTI2018-097723-B-I00), Gobierno de Aragón (Reference Group BSICoS T39-17R) by FEDER 2014-2020 and with a personal grant to S. Kontaxis, Programa Ibercaja-CAI de Estancias de Investigación (IT 16/18); Lithuanian Sports University, European Union's Framework Programme for Research and Innovation Horizon 2020 (2014-2020) under the Marie Skłodowska-Curie Grant Agreement No. 745755, and CIBER in Bioengineering, Biomaterials \& Nanomedicine (Instituto de Salud Carlos III).

\section{References}

[1] Crandall CG, and Gonzalez-Alonso J. Cardiovascular function in the heat-stressed humans. Acta Physiol 2010;199(4):407-423.

[2] Morrison SF. Central control of body temperature. F1000Res 2016; 5:880.

[3] Charkoudian N. Mechanisms and modifiers of reflex induced cutaneous vasodilation and vasoconstriction in humans. J Appl Physiol 2010;109(4):1221-1228.

[4] Hannuksela ML and Ellahham S. Benefits and risks of sauna bathing. Am J Med 2001;110(2):118-126.

[5] Malik M, et al. Heart rate variability: Standards of measurement, physiological interpretation, and clinical use. Eur Heart J 1996; 17(3):354-381.

[6] Bruce-Low SS, et al. Heart rate variability during high ambient heat exposure. Aviat Space Environ Med 2006;77(9):915-920.

[7] Zalewski P, et al. Cardiovascular and thermal response to dry-sauna exposure in healthy subjects. Physiol J 2014;2014:1-10.

[8] Hernando A, et al. Inclusion of respiratory frequency information in heart rate variability analysis for stress assessment. IEEE J Biomed Inform 2016;20(4):1016-1025.

[9] Martínez JP, et al. A wavelet-based ECG delineator: evaluation on standard databases. IEEE Trans Biomed Eng 2004;51(4):570-581.

[10] Mateo J and Laguna P. Analysis of heart rate variability in the presence of ectopic beats using the heart timing signal. IEEE Trans Biomed Eng 2003;50(3):334-343.

[11] Lázaro J, et al. Electrocardiogram derived respiratory rate from QRS slopes and R-wave angle. Ann Biomed Eng 2014; 42(10):2072-2083.

[12] Kontaxis S, et al. ECG-derived respiratory rate in atrial fibrillation. IEEE Trans Biomed Eng 2019;1-10.

[13] Bailón R, et al. The integral pulse frequency modulation model with time-varying threshold: application to heart rate variability analysis during exercise stress testing. IEEE Trans Biomed Eng 2011;58(3):642-652.

[14] Bailón R, et al. Analysis of heart rate variability using time-varying frequency bands based on respiratory frequency. Conf Proc IEEE Eng Med Biol Soc 2007;6674-6677.

[15] Hussain J and Cohen M. Clinical effects of regular dry sauna bathing: a systematic review. J Evid Based Complementary Altern Med 2018;2018:1-30.

Address for correspondence:

Spyridon Kontaxis (sikontax @unizar.es)

Campus Río Ebro, C/ María de Luna 1, 50018 Zaragoza, Spain 